\title{
An Interior Projected-Like Subgradient Method for Mixed Variational Inequalities
}

\author{
Guo-ji Tang' and Xing Wang ${ }^{2}$ \\ ${ }^{1}$ School of Science, Guangxi University for Nationalities, Nanning, Guangxi 530006, China \\ ${ }^{2}$ School of Information Technology, Jiangxi University of Finance and Economics, Nanchang 330013, China \\ Correspondence should be addressed to Guo-ji Tang; guojvtang@126.com
}

Received 5 February 2014; Accepted 12 March 2014; Published 15 May 2014

Academic Editor: Xian-Jun Long

Copyright (C) 2014 G.-j. Tang and X. Wang. This is an open access article distributed under the Creative Commons Attribution License, which permits unrestricted use, distribution, and reproduction in any medium, provided the original work is properly cited.

An interior projected-like subgradient method for mixed variational inequalities is proposed in finite dimensional spaces, which is based on using non-Euclidean projection-like operator. Under suitable assumptions, we prove that the sequence generated by the proposed method converges to a solution of the mixed variational inequality. Moreover, we give the convergence estimate of the method. The results presented in this paper generalize some recent results given in the literatures.

\section{Introduction}

Let $\mathbb{R}^{n}$ be endowed with the inner product $\langle\cdot, \cdot\rangle$ and the associated norm $\|\cdot\|$. Let $X$ be a nonempty, closed, and convex subset of $\mathbb{R}^{n}$ and let $F: \mathbb{R}^{n} \rightrightarrows \mathbb{R}^{n}$ be a set-valued mapping. Let $f: \mathbb{R}^{n} \rightarrow \mathbb{R} \cup\{+\infty\}$ be a proper, convex, and lower semicontinuous function. The mixed variational inequality problem (denoted by (MVI)) consists of finding an $x^{*} \in X$ such that there exists $u^{*} \in F\left(x^{*}\right)$ satisfying

$$
\left\langle u^{*}, x-x^{*}\right\rangle+f(x)-f\left(x^{*}\right) \geq 0, \quad \forall x \in X,
$$

which is well known to be a very useful tool to formulate a large class of problems encountered in mechanics, control, economics, structural engineering, social sciences, and so on [1-3]. In this paper, we denote by SOL(MVI) the solution set of (MVI).

It is well known that (MVI) includes a large variety of problems as special cases. For example, if $f=\delta_{X}$, where $\delta_{X}$ is the indicator function over the constraint set $X$, that is, $\delta_{X}(x)=0$ if $x \in X$ and $\delta_{X}(x)=+\infty$ otherwise, then (MVI) reduces to the generalized variational inequality (in short (GVI)): find an $x^{*} \in X$ such that there exists $u^{*} \in F\left(x^{*}\right)$ satisfying

$$
\left\langle u^{*}, x-x^{*}\right\rangle, \quad \forall x \in X .
$$

If $f=\delta_{X}$ and $F$ is single-valued, then (MVI) collapses to Stampacchia variational inequality problem: find $x^{*} \in X$ such that

$$
\left\langle F\left(x^{*}\right), x-x^{*}\right\rangle \geq 0, \quad \forall x \in X .
$$

One of the most interesting and important problems in the variational inequality theory is the development of an efficient iterative algorithm to compute approximate solutions, and the convergence analysis of the algorithm. Many methods have been proposed to solve (MVI) (see, e.g., [415]). Most of them are projection-type methods. Recently, projected subgradient methods have become effective and strong tools for solving (MVI) (see, e.g., $[8,14]$ ). However, all these methods are based on the Euclidean projection operator that produces iterates which hit the boundary of the constraints and might often lead to zigzagging effect resulting in slower convergence properties. Moreover, the projection itself can be computationally expensive, if the constraints are not simple.

Recently, to overcome the above difficulties, Auslender and Teboulle [16] proposed an approach for solving (GVI), which is to replace the classical projection with a nonEuclidean distance-like function that can automatically eliminate the constraints and produce interior trajectories. This 
line of analysis has been studied and developed over the recent years $[17,18]$.

On the other hand, unlike problems (2) and (3), in general, (MVI) is not equivalent to the fixed point problem involving the projection operator because of the presence of the nonlinear term $f$ in problem (MVI). However, the projection-like map introduced by Auslender and Teboulle [16-18] does not improve this situation. A natural problem is whether the techniques presented in [16] can be generalized from (GVI) to the setting of (MVI). That is we expect to devise a method for solving (MVI), which can not only inherit the nice property of interior-point methods of Auslender and Teboulle [16-18] but also overcome the difficulty as the presence of the nonlinear term $f$. This is the main motivation of this paper.

Motivated and inspired by the research work mentioned above, in this paper, we aim at extending the methods presented in [16] to mixed variational inequalities by introducing an interior projected-like subgradient method for mixed variational inequalities. The proposed method is based on using non-Euclidean projection-like operator. Under suitable assumptions, we prove that the sequence generated by the proposed method converges to a solution of the mixed variational inequality. Moreover, we give the convergence estimate of the method. The results presented in this paper generalize and improve some recent results.

\section{Preliminaries}

Definition 1. Let $F: \mathbb{R}^{n} \rightrightarrows \mathbb{R}^{n}$ be a set-valued mapping. Then the mapping $F$ is said to be

(i) monotone if, for any $x, y \in \mathbb{R}^{n}, u \in F(x)$, and $v \in$ $F(y)$,

$$
\langle v-u, y-x\rangle \geq 0
$$

(ii) maximal monotone if it is monotone and the graph of $F$, denoted by $\mathrm{Gph} F$, is not properly contained in the graph of any other monotone operator;

(iii) upper hemicontinuous at $x_{0} \in \mathbb{R}^{n}$ if, for any $y, z \in$ $\mathbb{R}^{n}$, the mapping $\varphi: \mathbb{R}_{+} \rightrightarrows \mathbb{R}, t \rightrightarrows\left\langle F\left(x_{0}+t y\right), z\right\rangle$ is upper continuous at $t=0$.

Remark 2. It is well known that $F: \mathbb{R}^{n} \rightrightarrows \mathbb{R}^{n}$ is maximal monotone if and only if

(i) for any $x \in \mathbb{R}^{n}, F(x)$ is a closed and convex subset of $\mathbb{R}^{n}$;

(ii) $F$ is upper hemicontinuous.

For many applications purposes, it will be useful to consider the ground set $X$ for (MVI) in the form

$$
X:=\bar{C} \cap V,
$$

where $C \subset \mathbb{R}^{n}$ is nonempty, open, and convex set with closure $\bar{C}$ and $V:=\left\{x \in \mathbb{R}^{n}: A x=b\right\}$, where $A: \mathbb{R}^{n} \rightarrow \mathbb{R}^{m}$ is a linear map, and $b \in \mathbb{R}^{m}$ (see, e.g., [16-18]).
Definition 3. Let $d: \mathbb{R}^{n} \times \mathbb{R}^{n} \rightarrow \mathbb{R}_{+} \cup\{+\infty\}$ be a proximal distance which for each $y \in C \cap V$ satisfies the following properties:

$\left(\mathrm{d}_{1}\right) d(\cdot, y)$ is proper, lower semicontinuous, and convex and $C^{1}$ on $C \cap V$, with $d(y, y)=0$ and $\nabla_{1} d(y, y)=$ 0 (the gradient of $d(\cdot, y)$ with respect to the first variable);

$\left(\mathrm{d}_{2}\right) \operatorname{dom} d(\cdot, y) \subset \bar{C}$, and $\operatorname{dom} \partial_{1} d(\cdot, y)=C$, where $\partial_{1} d(\cdot, y)$ denotes the subgradient map of the function $d(\cdot, y)$ with respect to the first variable;

$\left(\mathrm{d}_{3}\right) d(\cdot, y)$ is $\sigma$ strongly convex, over $C \cap V$; that is, there exists $\sigma>0$ such that for all $y \in C \cap V$,

$$
\begin{array}{r}
\left\langle\nabla_{1} d\left(x_{1}, y\right)-\nabla_{2} d\left(x_{2}, y\right), x_{1}-x_{2}\right\rangle \geq \sigma\left\|x_{1}-x_{2}\right\|^{2}, \\
\forall x_{1}, x_{2} \in C \cap V,
\end{array}
$$

for some norm $\|\cdot\|$ in $\mathbb{R}^{n}$.

We denote by $\mathscr{D}_{V}(C)$ the family of functions $d$ satisfying the above three properties.

Remark 4. It is easy to see that the usual squared Euclidean distance $d_{1}(x, y):=\|x-y\|^{2}$ satisfies the above three properties; that is, $d_{1}(\cdot, \cdot) \in \mathscr{D}_{V}(C)$. Therefore, the notion of proximal distance extends the usual squared Euclidean distance.

Given $d \in \mathscr{D}_{V}(C)$, it follows from the proof of Proposition 2.1 of [18] that for each $x \in C \cap V$ and for each $g \in \mathbb{R}^{n}$ there exists a unique (by strong convexity) point $p(g, x) \in$ $C \cap V$ solving

$$
p(g, x):=\underset{v \in V}{\operatorname{argmin}}\{\langle v, g\rangle+d(v, x)\} .
$$

From this fact, one can define a projected-like map as follows.

Definition 5. For any $g \in \mathbb{R}^{n}$, and any $x \in C \cap V$, a projectedlike map $p(\cdot, \cdot)$ is defined by

$$
p(g, x):=\underset{v \in V}{\operatorname{argmin}}\{\langle v, g\rangle+d(v, x)\} .
$$

Remark 6. (i) From the optimality conditions for the convex problem (8) (see, e.g., [19]), there exists $\mu:=\mu(g, x) \in \mathbb{R}^{m}$ such that

$$
g+A^{t} \mu+\nabla_{1} d(p(g, x), x)=0, \quad A p(g, x)=b .
$$

(ii) We would like to mention that the resulting projection-like map $p(g, x)$ remains in $C \cap V$, that is, an interior point with respect to the constraint set $C$. However, we also note that the properties of the map $p$ remain valid for an arbitrary closed and convex set $X$. The resulting projection map in that case leads to a noninterior projection-like map $p$ defined by

$$
p(g, x):=\underset{z \in X}{\operatorname{argmin}}\{\langle g, z\rangle+d(z, x)\}
$$


and characterized via

$$
\begin{aligned}
& \langle p(g, x)-\xi, g\rangle \\
& \quad \leq\left\langle\xi-p(g, x), \nabla_{1} d(p(g, x), x), x\right\rangle, \quad \forall \xi \in X .
\end{aligned}
$$

In particular for $d(x, y)=(1 / 2)\|x-y\|^{2}$, we have $p(g, x)=$ $P_{X}(x-g)$, where $P_{X}$ is the usual Euclidean projection operator.

Lemma 7 (Proposition 4.1 of [18]). For any $x \in C \cap V$ and any $g \in \mathbb{R}^{n}$ and $\lambda>0$, the point $p(\lambda g, x)$ satisfies $p(0, x)=x$ and the following properties hold:

(i) $\sigma\|x-p(\lambda g, x)\|^{2} \leq \lambda\langle x-p(\lambda g, x), g\rangle$;

(ii) $\|p(\lambda g, x)-x\| \leq \lambda \sigma^{-1}\|g\|$.

To establish convergence of our algorithm in this paper, for each given $d \in \mathscr{D}(C)$, we need a corresponding proximal distance $H$ satisfying some desirable properties.

Definition 8. Given $C \subset \mathbb{R}^{n}$, open and convex, and $d \in \mathscr{D}(C)$, a function $H: \mathbb{R}^{n} \times \mathbb{R}^{n} \rightarrow \mathbb{R}_{+} \cup\{+\infty\}$ is called the induced proximal distance to $d$ if $H$ is finite valued on $\bar{C} \times C$ such that for any $a, b \in C$,

$$
\begin{gathered}
H(a, a)=0, \\
\left\langle c-b, \nabla_{1} d(b, a)\right\rangle \leq H(c, a)-H(c, b), \quad \forall c \in \bar{C},
\end{gathered}
$$

and

(i) for any $y \in \bar{C}$ and $\left\{y_{k}\right\} \subset C$ being bounded with $\lim _{k \rightarrow+\infty} H\left(y, y_{k}\right)=0$, one has $\lim _{k \rightarrow+\infty} y_{k}=y$;

(ii) for any $y \in \bar{C}$ and $\left\{y_{k}\right\} \subset C$ converging to $y$, one has $\lim _{k \rightarrow+\infty} H\left(y, y_{k}\right)=0$;

(iii) for any $y \in \bar{C}, \lim _{x \in C,\|x\| \rightarrow \infty} H(y, x)=+\infty$.

We write $(d, H) \in \mathscr{F}_{+}(\bar{C})$ to quantify the triple $[\bar{C}, d, H]$ that satisfies the premises of Definition 8 .

Remark 9. One typical and useful example is the logarithmic quadratic distance $d$ given by

$$
d(z, x)=\sum_{j=1}^{n} x_{j}^{2} \omega\left(x_{j}^{-1} z_{j}\right)
$$

$$
\text { with } \omega(t)=\frac{\sigma}{2}(t-1)^{2}+\mu(t-\log t-1) \text {, }
$$

with $\sigma \geq \mu>0$. In that case, with $H(x, y)=\eta\|x-y\|^{2}, \eta:=$ $2^{-1}(\mu+\sigma)$, one can verify that $(d, H) \in \mathscr{F}_{+}(\bar{C})$ (see page 709 of [18]). For more examples, the interested reader is referred to [16-18].

\section{An Interior Projected-Like Subgradient Method}

In this paper, we adopt the following assumptions.

Assumption A. $\left(\mathrm{A}_{1}\right)$ The solution set of (MVI) is nonempty; that is, $\operatorname{SOL}(\mathrm{MVI}) \neq \emptyset$.

$\left(\mathrm{A}_{2}\right) \operatorname{dom} F:=\left\{x \in \mathbb{R}^{n}: F(x) \neq \emptyset\right\} \supset X$ and $F$ is maximal monotone.

$\left(\mathrm{A}_{3}\right) F$ is bounded on bounded subset of $\operatorname{ri}(X)$.

$\left(\mathrm{A}_{4}\right) f: \mathbb{R}^{n} \rightarrow \mathbb{R} \cup\{+\infty\}$ is convex, lower semicontinuous, proper, and finite on $X$.

$\left(A_{5}\right)$ The subdifferential map $\partial f$ is nonempty on $X$ and bounded on bounded subsets of $\operatorname{ri}(X)$.

Remark 10. The Assumptions $\left(\mathrm{A}_{1}\right)-\left(\mathrm{A}_{3}\right)$ are the same as Assumption $A$ of [16]. The Assumptions $\left(\mathrm{A}_{4}\right)-\left(\mathrm{A}_{5}\right)$ are the same as Assumptions $\left(B_{1}\right)-\left(B_{2}\right)$ of $[16]$. These assumptions are the same as those of [14], except for those on the mapping $F$.

Algorithm 11. Initialization. Let $x^{1} \in C \cap V$.

Iteration Step. Given $x^{k} \in C \cap V$, take $u^{k} \in F\left(x^{k}\right)$ and $v^{k} \in$ $\partial f\left(x^{k}\right)$, and compute

$$
x^{k+1}:=p\left(\lambda_{k}\left(u^{k}+v^{k}\right), x^{k}\right) \quad \text { for some } \lambda_{k}>0 .
$$

Remark 12. (i) If $f=\delta_{X}$, then (15) reduces to (3.11) of [16]. Thus, Algorithm 11 generalizes the basic iteration scheme of [16] from the variational inequality to the setting of the mixed variational inequality.

(ii) If $d(x, y)=(1 / 2)\|x-y\|^{2}$, then (15) becomes

$$
x^{k+1}:=P_{X}\left[x^{k}-\lambda_{k}\left(u^{k}+v^{k}\right)\right],
$$

which is the basic scheme of projected subgradient methods for mixed variational inequality (see, e.g., $[8,11,12,14]$ ).

We first establish the key result giving the main properties of the basic scheme (15) that will be used extensively to establish our convergence results.

Lemma 13. Let $(d, H) \in \mathscr{F}_{+}(\bar{C})$ and let $\left\{x^{k}\right\} \subset C \cap V$ be the sequence generated by Algorithm 11. Then the following properties hold:

(a) $\lambda_{k}\left\langle x^{k}-\xi, u^{k}+v^{k}\right\rangle \leq H\left(\xi, x^{k}\right)-H\left(\xi, x^{k+1}\right)+$ $\lambda_{k}^{2} \sigma^{-1}\left\|u^{k}+v^{k}\right\|^{2}, \quad \forall \xi \in \bar{C} \cap V ;$

(b) $H\left(x^{*}, x^{k+1}\right) \leq H\left(x^{*}, x^{k}\right)+\lambda_{k}^{2} \sigma^{-1}\left\|u^{k}+v^{k}\right\|^{2}, \forall x^{*} \in$ $S O L(M V I)$;

(c) for any $\xi \in \bar{C} \cap V$ and $u \in F(\xi)$,

$$
\begin{aligned}
\left\langle u, z^{l}-\xi\right\rangle+f\left(z^{l}\right)-f(\xi) & \\
\leq & \frac{H\left(\xi, x^{1}\right)+\sigma^{-1} \sum_{k=1}^{l} \lambda_{k}^{2}\left\|u^{k}+v^{k}\right\|^{2}}{s_{l}}
\end{aligned}
$$

$$
\text { with } z^{l}:=\sum_{k=1}^{l} \lambda_{k} x^{k} / s_{l} \text { and } s_{l}:=\sum_{k=1}^{l} \lambda_{k} \text {. }
$$


Proof. (a) Set $\alpha_{k}:=\left\langle u^{k}+v^{k}, x^{k}-x^{k+1}\right\rangle$. It follows from item (i) of Lemma 7 that

$$
\begin{aligned}
\sigma\left\|x^{k}-x^{k+1}\right\|^{2} & \leq \lambda_{k}\left\langle x^{k}-x^{k+1}, u^{k}+v^{k}\right\rangle \\
& \leq \lambda_{k} \alpha_{k} \leq \lambda_{k}\left\|x^{k}-x^{k+1}\right\| \cdot\left\|u^{k}+v^{k}\right\| .
\end{aligned}
$$

As a consequence, we have

$$
\begin{aligned}
& \alpha_{k} \geq 0, \\
& \left\|x^{k}-x^{k+1}\right\| \leq \lambda_{k} \sigma^{-1}\left\|u^{k}+v^{k}\right\|, \\
& \alpha_{k} \leq \lambda_{k} \sigma^{-1}\left\|u^{k}+v^{k}\right\|^{2} .
\end{aligned}
$$

Now let $\xi \in \bar{C} \cap V$. It follows from inequality (13) and the relation (9) that

$$
\lambda_{k}\left\langle x^{k+1}-\xi, u^{k}+v^{k}\right\rangle \leq H\left(\xi, x^{k}\right)-H\left(\xi, x^{k+1}\right) .
$$

Since

$$
\begin{aligned}
\left\langle u^{k}\right. & \left.+v^{k}, x^{k+1}-\xi\right\rangle \\
& =\left\langle u^{k}+v^{k}, x^{k+1}-x^{k}\right\rangle+\left\langle u^{k}+v^{k}, x^{k}-\xi\right\rangle,
\end{aligned}
$$

we have

$$
\begin{aligned}
& \lambda_{k}\left\langle x^{k}-\xi, u^{k}+v^{k}\right\rangle \\
& \quad \leq H\left(\xi, x^{k}\right)-H\left(\xi, x^{k+1}\right)+\lambda_{k} \alpha_{k}, \quad \forall \xi \in \bar{C} \cap V .
\end{aligned}
$$

This together with (19) implies item (a). have

(b) For each $x^{*} \in \operatorname{SOL}(\mathrm{MVI})$ with any $u^{*} \in F\left(x^{*}\right)$, we

$$
\begin{aligned}
& \left\langle x^{k}-x^{*}, u^{k}+v^{k}\right\rangle \\
& \geq\left\langle u^{k}, x^{k}-x^{*}\right\rangle+f\left(x^{k}\right)-f\left(x^{*}\right) \quad\left(\text { by } v^{k} \in \partial f\left(x^{k}\right)\right) \\
& \geq\left\langle u^{*}, x^{k}-x^{*}\right\rangle+f\left(x^{k}\right)-f\left(x^{*}\right)
\end{aligned}
$$

(by the monotonicity of $F$ )

$$
\left.\geq 0 \text {. (by } x^{*} \in \mathrm{SOL}(\mathrm{MVI})\right) \text {. }
$$

Combining with item (a) taking $\xi=x^{*}$, we obtain item (b).

(c) For each $\xi \in \bar{C} \cap V$, it follows from the monotonicity of $F, v^{k} \in \partial f\left(x^{k}\right)$ and item (a) that

$$
\begin{aligned}
& H\left(\xi, x^{k}\right)-H\left(\xi, x^{k+1}\right)+\lambda_{k}^{2} \sigma^{-1}\left\|u^{k}+v^{k}\right\|^{2} \\
& \geq \lambda_{k}\left\langle x^{k}-\xi, u^{k}+v^{k}\right\rangle \quad(\text { by item (a)) } \\
& \geq \lambda_{k}\left[\left\langle u^{k}, x^{k}-\xi\right\rangle+f\left(x^{k}\right)-f(\xi)\right] \quad\left(\text { by } v^{k} \in \partial f\left(x^{k}\right)\right) \\
& \geq \lambda_{k}\left[\left\langle u, x^{k}-\xi\right\rangle+f\left(x^{k}\right)-f(\xi)\right]
\end{aligned}
$$

$\forall u \in F(\xi) \quad$ (by the monotonicity of $F$ ).
Summing over $k=1, \ldots, l$ and dividing both members by $s_{l}$, by the convexity of $f$, one obtains that for each $u \in F(\xi)$,

$$
\begin{aligned}
& \left\langle u, z^{l}-\xi\right\rangle+f\left(z^{l}\right)-f(\xi) \\
& \quad \leq \frac{H\left(\xi, x^{1}\right)+\sigma^{-1} \sum_{k=1}^{l} \lambda_{k}^{2}\left\|u^{k}+v^{k}\right\|^{2}}{s_{l}} .
\end{aligned}
$$

This completes the proof.

From now on, we analyze the convergence behavior of Algorithm 11 by choosing the parameter $\lambda_{k}$ as

$$
\lambda_{k}=\theta_{k} \mu_{k}
$$

where the parameter $\theta_{k}$ is freely chosen and satisfies

$$
\theta_{k}>0, \quad \sum_{k=1}^{\infty} \theta_{k}=+\infty, \quad \sum_{k=1}^{\infty} \theta_{k}^{2}<+\infty .
$$

About the parameter $\mu_{k}$, we make the following assumptions. Assumption B. $\left(\mathrm{B}_{1}\right)\left\{u^{k}\right\}$ and $\left\{v^{k}\right\}$ are bounded $\Rightarrow$ there exists $\mu_{*}>0$ such that $\mu_{k} \geq \mu_{*}$ for all $k$; all $k$.

$\left(\mathrm{B}_{2}\right)$ there exits some $L>0$ such that $\mu_{k}\left\|u^{k}+v^{k}\right\| \leq L$ for

Remark 14. We would like to mention that there are many well-known alternatives for the choice of $\mu_{k}$; for example,

$$
\begin{gathered}
\mu_{k}=\left\|u^{k}+v^{k}\right\|^{-1}, \\
\mu_{k}=\max \left\{1,\left\|u^{k}+v^{k}\right\|^{-1}\right\}, \\
\mu_{k}=\left(1+\left\|u^{k}+v^{k}\right\|\right)^{-1}, \\
\mu_{k}=\max \left\{\theta_{k},\left\|u^{k}+v^{k}\right\|^{-1}\right\} .
\end{gathered}
$$

The interested reader is referred to $[8,14,20,21]$. Note that both hypotheses (with $L=1$ for $\left(\mathrm{B}_{2}\right)$ ) are satisfied by all the above suggested choices for $\mu_{k}$. In particular, if $d(x, y)=$ $(1 / 2)\|x-y\|^{2}$ and $\lambda_{k}=\theta_{k} \mu_{k}$ with $\mu_{k}=\left(\max \left\{1,\left\|u^{k}\right\|+\right.\right.$ $\left.\left.\left\|v^{k}\right\|\right\}\right)^{-1}$, then Algorithm 11 reduces to the method proposed by Xia et al. [14].

Theorem 15. Let $(d, H) \in \mathscr{F}_{+}(\bar{C})$. Suppose that Assumptions $A$ and $B$ hold. Let $\left\{x^{l}\right\}$ be the sequence generated by Algorithm 11, and set

$$
s_{l}=\sum_{k=1}^{l} \lambda_{k}, \quad z^{l}=\frac{\sum_{k=1}^{l} \lambda_{k} x^{k}}{s_{l}} .
$$

Then the sequences $\left\{x^{l}\right\},\left\{z^{l}\right\}$ are bounded and each cluster point of the sequence $\left\{z^{l}\right\}$ belongs to the solution set of (MVI). Moreover, suppose that

$$
H(x, y)=v\|x-y\|^{2}, \quad \text { for some } v>0 .
$$

Then the whole sequence $\left\{z^{l}\right\}$ converges to some solution of (MVI). 
Proof. We divide the proof into two steps.

Step 1. Invoking item (b) of Lemma 13 , and using assumption $\left(\mathrm{B}_{2}\right)$, we get by induction

$$
\begin{aligned}
& H\left(x^{*}, x^{k}\right) \\
& \quad \leq H\left(x^{*}, x^{1}\right)+L^{2} \sigma^{-1} \sum_{l=1}^{k} \theta_{l}^{2}, \quad \forall x^{*} \in \operatorname{SOL}(\mathrm{MVI}) .
\end{aligned}
$$

Using $\sum_{k=1}^{\infty} \theta_{k}^{2}<+\infty$, it is easy to see that $\left\{H\left(x^{*}, x^{k}\right)\right\}$ is bounded. From item (iii) of Definition 8 , we know that $\left\{x^{k}\right\}$ is bounded. As a consequence the sequence $\left\{z^{k}\right\}$ is bounded. It follows from Assumptions $\left(A_{3}\right)$ and $\left(A_{5}\right)$ that the sequences $\left\{u^{k}\right\}$ and $\left\{v^{k}\right\}$ are also bounded. Then, using Assumption $\left(B_{1}\right)$ we get

$$
\mu_{k} \geq \mu_{*}, \quad \forall k .
$$

Since $s_{l}=\sum_{k=1}^{l} \theta_{k} \mu_{k}$, using inequality (32), one has $s_{l} \geq$ $\mu_{*} \sum_{k=1}^{l} \theta_{k}$. It follows from item (c) of Lemma 13 that for any $\xi \in \bar{C} \cap V$ and $u_{\xi} \in F(\xi)$,

$$
\begin{aligned}
& \left\langle u_{\xi}, z^{l}-\xi\right\rangle+f\left(z^{l}\right)-f(\xi) \leq \frac{K_{\xi}}{\sum_{k=1}^{l} \theta_{k}}, \\
& \text { with } K_{\xi}=\frac{H\left(\xi, x^{1}\right)+\sigma^{-1} L^{2} \sum_{k=1}^{l} \theta_{k}^{2}}{\mu_{*}} .
\end{aligned}
$$

Let $z^{\infty}$ be a cluster point of the sequence $\left\{z^{l}\right\}$. Since $\sum_{k=1}^{\infty}=$ $+\infty$ and $f$ is lower semicontinuous, taking limits in both sides of inequality (32), one gets

$$
\begin{array}{r}
\left\langle u_{\xi}, z^{\infty}-\xi\right\rangle+f\left(z^{\infty}\right)-f(\xi) \leq 0, \\
\forall \xi \in X=\bar{C} \cap V, \quad \forall u_{\xi} \in F(\xi) .
\end{array}
$$

Furthermore, $z^{\infty} \in X$. Now set $F_{p}(x):=F(x)+\partial\left(f+\delta_{X}\right)(x)$, where $\delta_{X}$ denotes the indicator function over $X$; that is, if $x \in$ $X$, then $\delta_{X}=0$, otherwise, $\delta_{X}=+\infty$. Since $F(x)$ is nonempty for each $x \in X$, it follows that $F_{p}$ is maximal monotone. From the definition of subdifferential, we have

$$
\begin{aligned}
\left\langle v_{\xi}, z^{\infty}-\xi\right\rangle+f(\xi)-f\left(z^{\infty}\right) \leq 0, \\
\forall v_{\xi} \in \partial\left(f+\delta_{X}\right)(\xi), \quad \forall \xi \in X .
\end{aligned}
$$

Summing both sides of inequalities (34) and (35), we obtain

$$
\left\langle u_{\xi}+v_{\xi}, z^{\infty}-\xi\right\rangle \leq 0, \quad \forall \xi \in X, \forall g_{p}:=u_{\xi}+v_{\xi} \in F_{p}(\xi) .
$$

Since $F_{p}$ is maximal monotone, this means that $0 \in F_{p}\left(z^{\infty}\right)$, which is equivalent to say that $z^{\infty}$ is a solution of (MVI).

Step 2. Invoking item (b) of Lemma 13, and using Assumption $\left(\mathrm{B}_{2}\right)$, we get by induction

$$
\begin{array}{r}
H\left(x^{*}, x^{k+p}\right) \leq H\left(x^{*}, x^{k}\right)+\sigma^{-1} L^{2} \sum_{l=k}^{k+p} \theta_{l}^{2}, \\
\forall x^{*} \in \operatorname{SOL}(\mathrm{MVI}), \quad \forall p \in \mathbb{N} .
\end{array}
$$

Using $\sum_{k=1}^{\infty} \theta_{k}^{2}<+\infty$ and (30) we get

$$
\begin{array}{r}
\left\|x^{k+p}-x^{*}\right\|^{2} \leq\left\|x^{k}-x^{*}\right\|^{2}+\rho_{k}, \quad \forall x^{*} \in \operatorname{SOL}(\mathrm{MVI}), \\
\text { with } \lim _{k \rightarrow \infty} \rho_{k}=0 .
\end{array}
$$

From item (i), we know that the sequence $\left\{z^{l}\right\}$ is bounded with all its cluster points belonging to SOL(MVI). Thus, to complete the proof of our claim on the whole convergence of the sequence $\left\{z^{l}\right\}$ to a solution of (MVI), we only need to prove that the sequence $\left\{z^{l}\right\}$ has a unique cluster point. The proof of the remainder is exactly the same one as given by Bruck [22] in Steps 2 and 3 of Theorem 1. For the sake of convenience, the reader is also referred to Corollary 1 of the recent paper [16] (page 38-40) and so we omit it here.

Remark 16. If $f=\delta_{X}$, then Theorem 15 reduces to item (a) of Theorem 1 and Corollary 1 of [16]. Thus, we extend the main results of [16] from variational inequalities to the setting of mixed variational inequalities.

Remark 17. Compared with Theorem 3.5 of Xia et al. [14], Theorem 15 says that the sequence $\left\{z^{l}\right\}$, rather than $\left\{x^{k}\right\}$, is convergent to a solution of (MVI).

Definition 18. A function $\gamma: X \subset \mathbb{R}^{n} \rightarrow \mathbb{R}_{+}$is called a gap function for (MVI) when the following statements hold:

(i) $\gamma(z) \geq 0$ for any $z \in X$;

(ii) $\gamma\left(z_{0}\right)=0$ if and only if $z_{0}$ is the solution of (MVI).

Clearly, a gap function with the properties of Definition 18 allows us to reformulate (MVI) as an optimization problem, namely, as

$$
\min _{z \in X} \gamma(z) .
$$

In order to establish efficiency estimates for mixed variational inequality problems, we will introduce a gap function for (MVI).

Proposition 19. The function

$$
\gamma(z)=\sup \{\langle g, z-\xi\rangle+f(z)-f(\xi): g \in F(\xi), \xi \in X\}
$$

is a gap function for (MVI).

Proof. The proof is in two parts.

(i) $[\gamma(z) \geq 0]$ It is easy to see that

$$
\begin{aligned}
\gamma(z) & \\
& =\sup \{\langle g, z-x\rangle+f(z)-f(x): g \in F(x), x \in X\} \\
& \geq\left\langle g_{z}, z-z\right\rangle+f(z)-f(z) \quad\left(g_{z} \in F(z)\right) \\
& =0 .
\end{aligned}
$$


(ii) $\left[\gamma\left(z_{0}\right)=0 \Leftrightarrow z_{0} \in \operatorname{SOL}(\mathrm{MVI})\right]$ If $z_{0} \in X$ solves (MVI), then there exists $g_{z_{0}} \in F\left(z_{0}\right)$ such that

$$
\left\langle g_{z_{0}}, x-z_{0}\right\rangle+f(x)-f\left(z_{0}\right) \geq 0, \quad \forall x \in X
$$

By monotonicity of $F$, we have

$$
\begin{array}{r}
\left\langle g_{x}, x-z_{0}\right\rangle+f(x)-f\left(z_{0}\right) \geq 0, \\
\forall x \in X, \quad g_{x} \in F(x) .
\end{array}
$$

Therefore, it follows that

$$
\begin{aligned}
& \gamma\left(z_{0}\right) \\
& =\sup \left\{\left\langle g_{x}, z_{0}-x\right\rangle+f\left(z_{0}\right)-f(x): g_{x} \in F(x), x \in X\right\} \\
& \leq 0 .
\end{aligned}
$$

By item (i), we have $\gamma\left(z_{0}\right)=0$. get

Conversely, if $\gamma\left(z_{0}\right)=0$, then, by the definition of $\gamma$, we

$$
\left\langle g_{x}, x-z_{0}\right\rangle+f(x)-f\left(z_{0}\right) \geq 0, \quad \forall x \in X, g_{x} \in F(x) .
$$

By maximal monotonicity of $F$, we know that $F$ is upper hemicontinuous. Therefore, it is easy to see that there exists $g_{z_{0}} \in F\left(z_{0}\right)$ such that

$$
\left\langle g_{z_{0}}, y-z_{0}\right\rangle+f(y)-f\left(z_{0}\right) \geq 0, \quad \forall y \in X
$$

This completes the proof.

In order to characterize the convergence estimate of Algorithm 11, we also need the quantity

$$
V_{H}=\sup \left\{H\left(\xi, x^{1}\right): \xi \in X\right\} \text {. }
$$

Now we present the convergence estimate of Algorithm 11.

Theorem 20. Let $(d, H) \in \mathscr{F}_{+}(\bar{C})$. Suppose that Assumptions $A$ and $B$ hold. Let $\left\{x^{l}\right\}$ be the sequence generated by Algorithm 11 and set

$$
s_{l}=\sum_{k=1}^{l} \lambda_{k}, \quad z^{l}=\frac{\sum_{k=1}^{l} \lambda_{k} x^{k}}{s_{l}} .
$$

If $V_{H}$ is finite, then we have

$$
\gamma\left(z^{l}\right) \leq\left(\frac{V_{H}+\sigma^{-1} L^{2} \sum_{k=1}^{\infty} \theta_{k}^{2}}{\mu_{*}}\right) \cdot \frac{1}{\sum_{k=1}^{l} \theta_{k}}, \quad \forall l .
$$

Proof. If $V_{H}$ is finite, then the estimate (49) is an immediate consequence of inequality (33) and the definition of the gap function $\gamma(\cdot)$.

Remark 21. If $f=\delta_{X}$, then Theorem 20 reduces to item (b) of Theorem 1 of [16].

\section{Conflict of Interests}

The authors declare that there is no conflict of interests regarding the publication of this paper.

\section{Acknowledgments}

This research is supported by Guangxi Natural Science Foundation (2013GXNSFBA019015), Scientific Research Foundation of Guangxi University for Nationalities (2012QD015), and Open Fund of Guangxi Key Laboratory of Hybrid Computation and IC Design Analysis (2013HCIC08).

\section{References}

[1] W. Han and B. D. Reddy, "On the finite element method for mixed variational inequalities arising in elastoplasticity," SIAM Journal on Numerical Analysis, vol. 32, no. 6, pp. 1778-1807, 1995.

[2] F. Giannessi, A. Maugeri, and P. M. Pardalos, Equilibrium Problems and Variational Models, Kluwer Academic Publishers, Boston, Mass, USA, 2001.

[3] I. V. Konnov and E. O. Volotskaya, "Mixed variational inequalities and economic equilibrium problems," Journal of Applied Mathematics, vol. 2, no. 6, pp. 289-314, 2002.

[4] Q. H. Ansari and J. C. Yao, "Iterative schemes for solving mixed variational-like inequalities," Journal of Optimization Theory and Applications, vol. 108, no. 3, pp. 527-541, 2001.

[5] A. Bnouhachem, M. Li, M. Khalfaoui, and S. Zhaohan, "A modified inexact implicit method for mixed variational inequalities," Journal of Computational and Applied Mathematics, vol. 234, no. 12, pp. 3356-3365, 2010.

[6] I. V. Konnov and S. Kum, "Descent methods for mixed variational inequalities in a Hilbert space," Nonlinear Analysis, vol. 47, pp. 561-572, 2001.

[7] I. V. Konnov, "A combined relaxation method for a class of nonlinear variational inequalities," Optimization, vol. 51, no. 1, pp. 127-143, 2002.

[8] P.-E. Maingé, "Projected subgradient techniques and viscosity methods for optimization with variational inequality constraints," European Journal of Operational Research, vol. 205, no. 3, pp. 501-506, 2010.

[9] M. A. Noor, "Proximal methods for mixed variational inequalities," Journal of Optimization Theory and Applications, vol. 115, no. 2, pp. 447-452, 2002.

[10] O. V. Pinyagina and M. S. S. Ali, "Descent method for monotone mixed variational inequalities," Calcolo, vol. 45, no. 1, pp. 1-15, 2008.

[11] G.-J. Tang and N.-J. Huang, "Projected subgradient method for non-Lipschitz set-valued mixed variational inequalities," Applied Mathematics and Mechanics. English Edition, vol. 32, no. 10, pp. 1345-1356, 2011.

[12] G. J. Tang and N. J. Huang, "Strong convergence of an inexact projected subgradient method for mixed variational inequalities," Optimization, 2012.

[13] S. L. Wang, H. Yang, and B. S. He, "Inexact implicit method with variable parameter for mixed monotone variational inequalities," Journal of Optimization Theory and Applications, vol. 111, no. 2, pp. 431-443, 2001.

[14] F.-Q. Xia, N.-J. Huang, and Z.-B. Liu, "A projected subgradient method for solving generalized mixed variational inequalities," Operations Research Letters, vol. 36, no. 5, pp. 637-642, 2008. 
[15] L.-C. Zeng and J.-C. Yao, "Convergence analysis of a modified inexact implicit method for general mixed monotone variational inequalities," Mathematical Methods of Operations Research, vol. 62, no. 2, pp. 211-224, 2005.

[16] A. Auslender and M. Teboulle, "Projected subgradient methods with non-Euclidean distances for non-differentiable convex minimization and variational inequalities," Mathematical Programming, vol. 120, no. 1, pp. 27-48, 2009.

[17] A. Auslender and M. Teboulle, "Interior projection-like methods for monotone variational inequalities," Mathematical Programming, vol. 104, no. 1, pp. 39-68, 2005.

[18] A. Auslender and M. Teboulle, "Interior gradient and proximal methods for convex and conic optimization," SIAM Journal on Optimization, vol. 16, no. 3, pp. 697-725, 2006.

[19] R. T. Rockafellar, Convex Analysis, Princeton University Press, Princeton, NJ, USA, 1970.

[20] Ya. I. Alber, A. N. Iusem, and M. V. Solodov, "On the projected subgradient method for nonsmooth convex optimization in a Hilbert space," Mathematical Programming, vol. 81, no. 1, pp. 2335, 1998.

[21] Ya. I. Alber and A. N. Iusem, "Extension of subgradient techniques for nonsmooth optimization in Banach spaces," SetValued Analysis, vol. 9, no. 4, pp. 315-335, 2001.

[22] R. E. Bruck Jr., "On the weak convergence of an ergodic iteration for the solution of variational inequalities for monotone operators in Hilbert space," Journal of Mathematical Analysis and Applications, vol. 61, no. 1, pp. 159-164, 1977. 


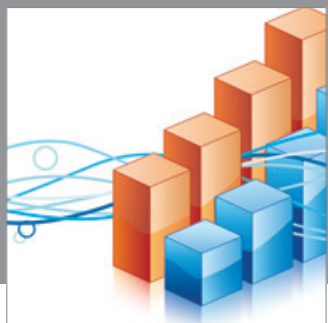

Advances in

Operations Research

mansans

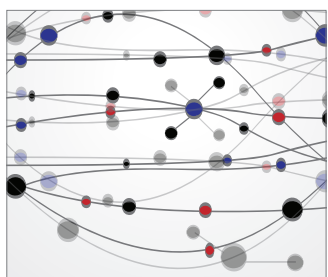

The Scientific World Journal
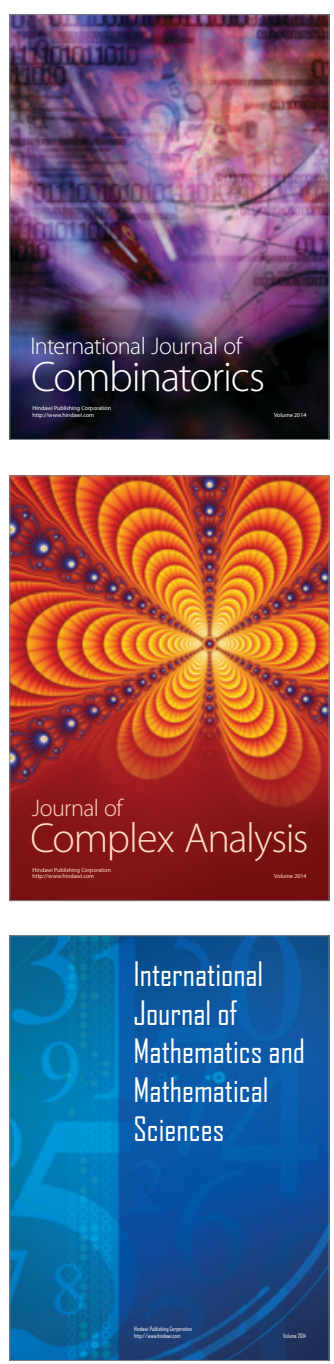
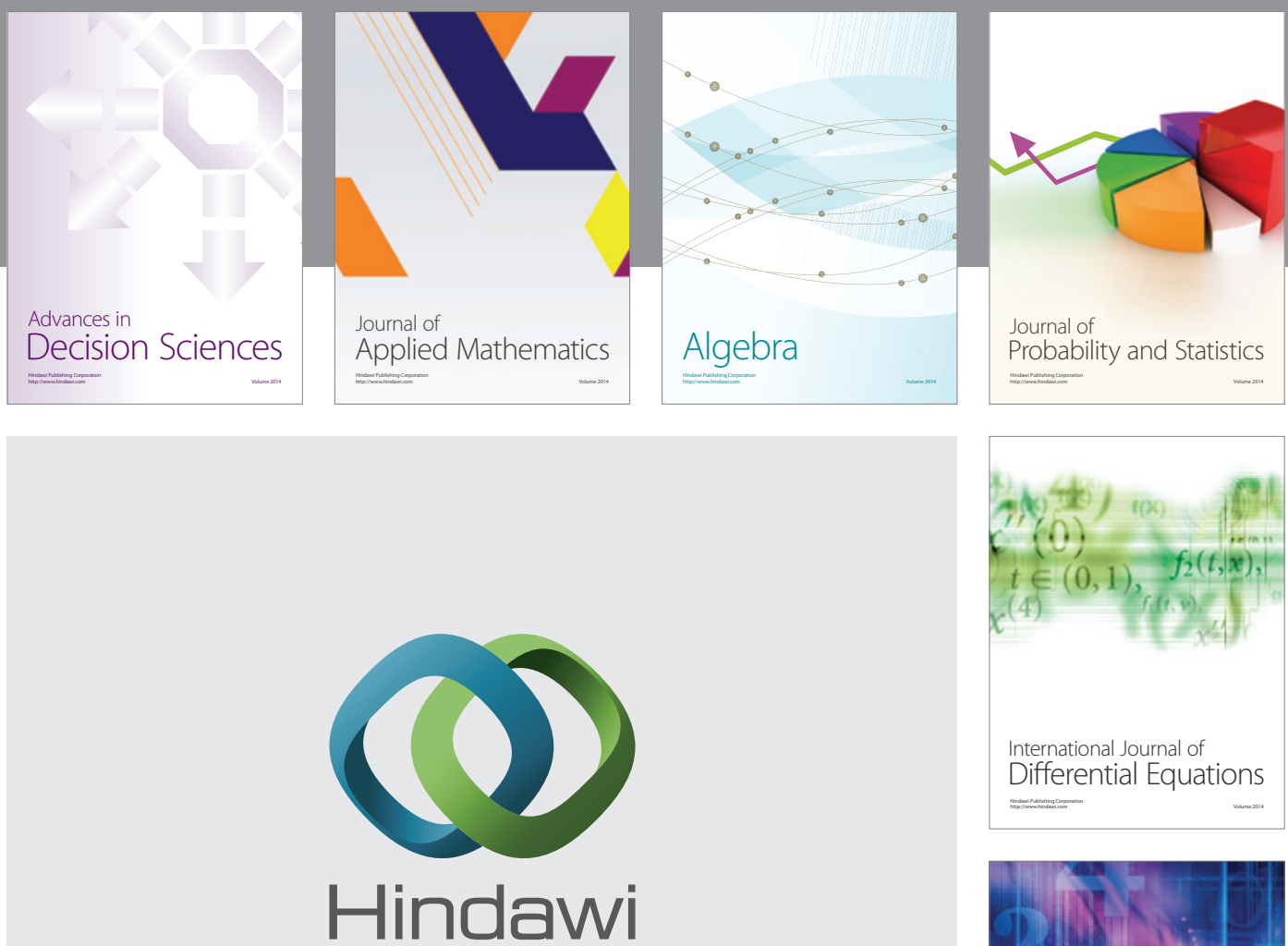

Submit your manuscripts at http://www.hindawi.com
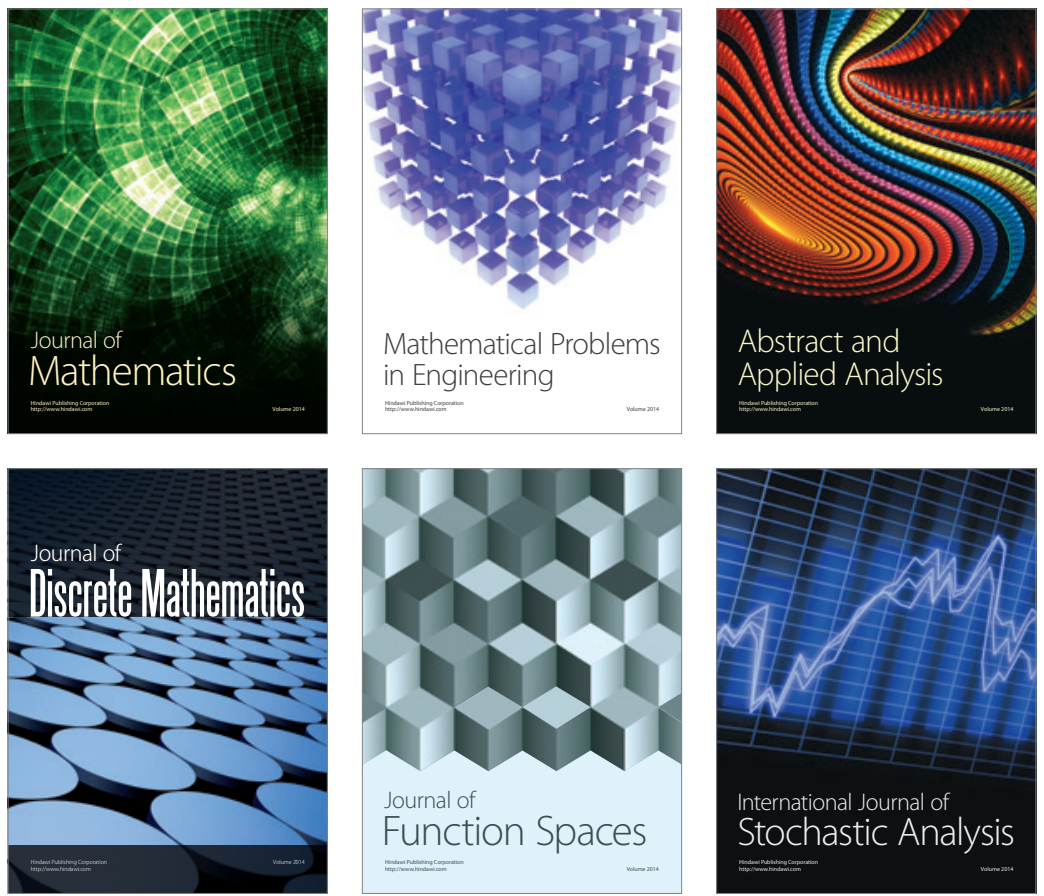

Journal of

Function Spaces

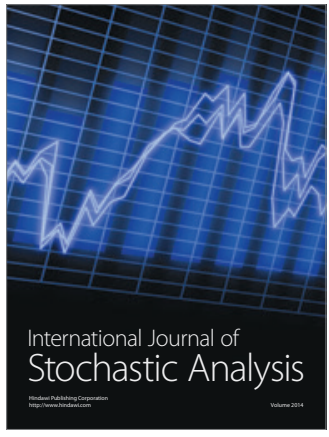

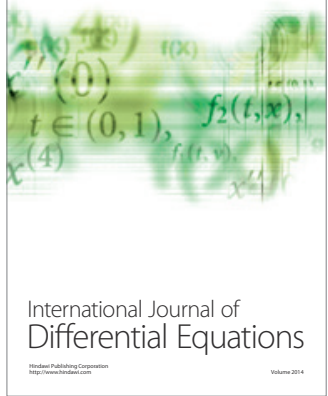
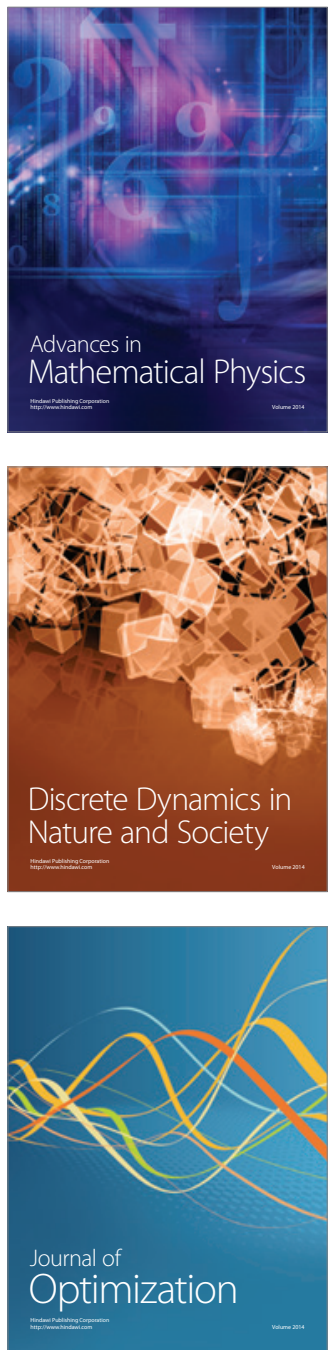